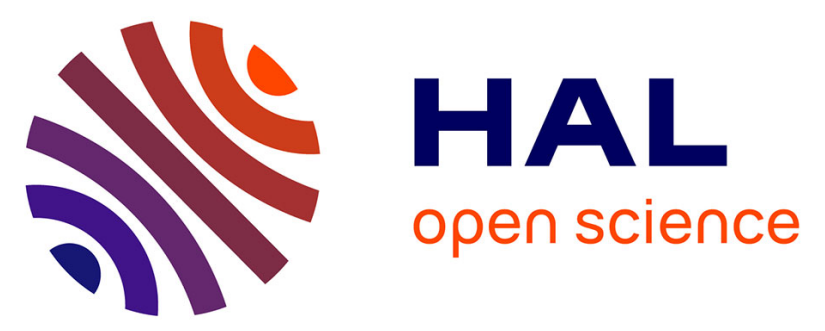

\title{
Value of 3D right ventricular function over 2D assessment in acute pulmonary embolism
}

Pamela Moceri, Nicolas Duchateau, Benjamin Sartre, Delphine Baudouy, Fabien Squara, Maxime Sermesant, Emile Ferrari

\section{- To cite this version:}

Pamela Moceri, Nicolas Duchateau, Benjamin Sartre, Delphine Baudouy, Fabien Squara, et al.. Value of 3D right ventricular function over 2D assessment in acute pulmonary embolism. Echocardiography, 2021, 10.1111/echo.15167 . hal-03544761

\section{HAL Id: hal-03544761 \\ https://hal.science/hal-03544761}

Submitted on 26 Jan 2022

HAL is a multi-disciplinary open access archive for the deposit and dissemination of scientific research documents, whether they are published or not. The documents may come from teaching and research institutions in France or abroad, or from public or private research centers.
L'archive ouverte pluridisciplinaire HAL, est destinée au dépôt et à la diffusion de documents scientifiques de niveau recherche, publiés ou non, émanant des établissements d'enseignement et de recherche français ou étrangers, des laboratoires publics ou privés. 


\section{Value of 3D right ventricular function over 2D assessment in acute pulmonary embolism}

Short title : 3D RV Function in pulmonary embolism

Pamela Moceri MD, $\mathrm{PhD}^{+, \neq, \text {; }}$ Nicolas Duchateau PhDl'; Benjamin Sartre MD; Delphine Baudouy MD, PhD ; Fabien Squara MD;; Maxime Sermesant $\mathrm{PhD}^{+*}$; Emile Ferrari $\mathrm{MD}^{\ddagger *}$.

† Université Côte d'Azur, Inria Epione team, Sophia Antipolis, France.

‡ Centre Hospitalier Universitaire de Nice, Service de Cardiologie, Nice, France.

§ UR2CA, Université Côte d'Azur, Nice, France.

| | Creatis, CNRS UMR5220, INSERM U1206, Université Lyon 1, France.

* EF and MS co-share senior authorship

Manuscript word count: 2995

Corresponding author: Dr Pamela Moceri, CHU de Nice - Hôpital Pasteur 1 Avenue de la voie romaine, CS 51069 - 06001 Nice, France.

Email: moceri.p@chu-nice.fr Tel : +33492037734 - Fax: +33492037876

Conflict of interest: None

Author contributions: PM, ND, BS, DB, FS, MS and EF contributed to the study design, data collection, data analysis or interpretation, critical revision - approval of the manuscript. PM, BS, ND and MS also contributed to drafting the article, statistics and funding the study. 


\section{ABSTRACT}

Background: Pulmonary embolism (PE) is a common life-threatening disease, with mortality related to right ventricular (RV) dysfunction.

Aims: To investigate the value of 3D global and regional right ventricular strain in patients with acute PE and at 1 month, as compared to a control population.

Methods and Results: We conducted a longitudinal case-control prospective study, including 24 consecutive intermediate-risk PE patients. All patients underwent $2 \mathrm{D}$ and $3 \mathrm{D}$ transthoracic echocardiography within 12 hours of PE diagnosis and 1 month after hospital discharge. A control group was recruited, consisting of healthy volunteers matched on age and sex with PE patients. 3D RV echocardiographic sequences were analysed by commercial RV-specific software and output meshes were postprocessed to extract regional deformation. 3D echocardiographic 1-month follow-up was available in 18 patients. During acute $\mathrm{PE}$, area strain was substantially altered in the RV free wall and within the trabecular septum. PE patients initially had RV dysfunction as assessed by 2D and 3D parameters. At follow-up, 2D parameters were restored compared to the control group, contrary to 3D RV area and circumferential strains. The McConnell's sign was identified in $83 \%$ of patients and was associated with reduced apical and global RV area strain.

Conclusions: Our 3D RV strain study demonstrates an incomplete recovery of 3D strain parameters 1 month after an episode of intermediate-risk acute PE despite restored 2D parameters. Further studies are required to assess the prognostic role and implications of this residual RV strain impairment after PE.

KEYWORDS: pulmonary embolism; right ventricular function; 3D echocardiography; speckle-tracking_ 


\section{INTRODUCTION}

Pulmonary embolism is defined as the migration into the pulmonary artery tree of an embolus responsible for the obliteration of the trunk, or one or more branches of the pulmonary artery. The estimated incidence of pulmonary embolism in the general population is $60-70$ cases per 100.000 people [1]. The obstruction of the pulmonary tree may lead to right ventricular (RV) dysfunction, as assessed with echocardiography [2,3]. In addition to standard parameters, RV strain assessment, especially using three-dimensional (3D) echocardiography, may be of interest $[4,5]$ in patients with .

We wanted to investigate the RV adaptation to acute pulmonary embolism and its recovery at one month after hospitalisation. Thus, the aim of this study is to compare 3D RV strain parameters and 2D classic RV parameters in patients with intermediate-high risk pulmonary embolism at the time of acute pulmonary embolism, after 1 month post discharge and to compare patients to a sex- and age-matched control population. We also aimed to compare 3D RV strain in acute pulmonary embolism patients with or without Mc-Connell's signs. 


\section{METHODS}

\section{Study design and patients}

We performed a prospective case control and longitudinal study on adult patients hospitalized for acute pulmonary embolism (PE) in our centre (Cardiology department, CHU de Nice, FR) between May 2017 and March 2018. Consecutive PE patients were included if they were intermediate-high risk PE adult patients according to the ESC guidelines[6] (Positive cardiac biomarkers; BNP and/or Troponin; and evidence of RV dysfunction, no shock or hypotension) with no previous known significant cardiac or respiratory condition). Patients were enrolled into a standardized echocardiographic protocol including 2D and 3D echocardiography on admission and after 1 month (end of the study). The first echocardiography was performed within 12 hours from PE diagnosis. Our study protocol is in compliance with the declaration of Helsinki and was approved by the local research Ethics committee. Personal patient consent was obtained from each participant.

Volunteers without any significant respiratory or cardiac condition and matched for age and sex to the patients' group were recruited from the outpatient clinic to serve as controls. Inclusion criteria were a normal transthoracic echocardiography and sinus rhythm.

Demographics and clinical data (age, gender, height, weight, peak BNP and troponin, NYHA class) were collected on inclusion and after 1 month.

\section{D echocardiographic acquisitions and measurements}

Echocardiographic examination was performed using an EPIQ-7 ultrasound system and an X5-1 transducer (Philips Medical system, Andover, MA). Doppler echocardiography was performed according to the recommendations of the American Society of Echocardiography (ASE) / European Association for Cardiovascular Imaging (EACVI) $[2,7]$.

Two cardiologists with advanced training in echocardiography performed the cine-loop acquisitions and interpreted the 2D-echo datasets. The following parameters were measured and averaged over 
three consecutive cycles: left ventricular (LV) ejection fraction, right atrial pressure according to the inferior vena cava dimension and collapsibility, systolic pulmonary artery pressure, tricuspid annular peak systolic velocity $\left(s^{\prime}\right)$, tricuspid annular plane systolic excursion (TAPSE), RV basal diameter, RV/LV diameter ratio, RV fractional area change, presence of paradoxical septum motion. The presence of the McConnell's sign, defined by regional RV wall motion abnormality of the basal and mid free wall with apical RV hyper contractility[8], was also assessed.

\section{D Transthoracic Echocardiography}

At least four 3D cine-loops of the RV were acquired from an apical 4chamber view focused on the RV, using an EPIQ-7 ultrasound system and a matrix-array X5-1 transducer (Philips Medical system, Andover, MA). Fullvolume acquisition over 2 heartbeats (for loop storage) was performed using ECG-gating over 4 cardiac cycles, during a breath-hold if possible. Volume rate was maximized to allow the use of the RV-dedicated quantification software (4D RV Function 2.0, TomTec Imaging Systems $\mathrm{GmbH}, \mathrm{DE}$ ) and follow the current quantification guidelines (frame rate $>20 \mathrm{~Hz}$ [9]. Care was taken to include the entire $\mathrm{RV}$ within the images. Digital 3D sequences were stored and analysed using the RV quantification software. This software allowed semi-automatic delineation of the RV endocardium, which was subsequently tracked along the cardiac cycle using 3D speckle-tracking. It directly provided an estimation of the RV end-diastolic and end-systolic volumes, ejection fraction, and wallspecific peak strain.

\section{Deformation analysis}

RV surface meshes along the cardiac cycle were exported from the commercial software and post-processed. Local motion and deformation were computed at each point of the RV mesh using VTK (v7.10, Kitware, New York, US), similar to our previous work[9]. Circumferential and longitudinal strains computations were based on the engineering strain --the relative change of length from end-diastole--- along these directions, 
within a $5 \mathrm{~mm}$ neighbourhood. Area strain was computed as the area change of each mesh triangle with respect to end-diastole. Our results focus on end-systolic strain values. Spatial correspondences between subjects' data were obtained by using the IDs of the mesh points provided by the commercial software, which are consistent between subjects. Temporal correspondences were achieved by normalizing the data according to the cardiac cycle. For regional analysis, the RV free wall and septum were both labelled according to 4 regions: inlet, outlet, body and apex[10] (Figure 1). Finally, the statistical results over a given subgroup were displayed on its average mesh, obtained by standard computational anatomy tools (Procrustes analysis). These computations were carried out in VTK and Matlab (v.R2011a, MathWorks, Natick, US).

\section{Statistical analysis}

Data were summarized as mean \pm standard deviation for continuous variables with normal distribution; median [95\% confidence interval] for other continuous variables and number of subjects (\%) for categorical variables. Comparisons between PE patients on admission and after 1 month were tested by the paired Student t-test for normally distributed variables or by the Wilcoxon test otherwise. Comparisons between PE patients and controls were performed using the Student t-test for normally distributed variables or by the Mann-Whitney U-test otherwise. Categorical variables were compared using Fisher's exact test. A ROC curve was built to assess the relationship between McConnell's sign and the RV apical area strain in the whole population. These analyses were performed using MedCalc 17.5.5 (MedCalc Software, Mariakerke, BE). In all analyses, statistical significance was defined as a p-value $<0.05$.

\section{RESULTS}

\section{General characteristics of the population}

Twenty-four patients with acute intermediate-high risk of early mortality PE were included. One patient died from cerebral haemorrhage 
in a context of unknown glioblastoma before the follow-up. Another patient was lost to follow-up. In four patients, the 3D echocardiographic data were not suitable for quantification with the commercial software due to limited image quality.

Our patient population was predominantly composed by female $(55.6 \%)$ aged of $75.5 \pm 10.4$ years. Mean BNP on inclusion was $445.8 \pm 282.5 \mathrm{pg} / \mathrm{ml}$ and mean troponin was $1083.6 \pm 560.1 \mathrm{ng} / \mathrm{ml}$. All patients had intermediate-high risk PE. No significant difference of age and sex was observed between patients and controls $(p=0.47$ and 0.96 , respectively).

\section{Two-dimensional RV function}

The 2D ultrasound baseline and follow-up characteristics of our final cohort are summarized in Table 1 . Systolic pulmonary artery pressures decreased significantly in patients after 1 month of therapy, from $52.2 \pm 18.5 \mathrm{mmHg}$ to $41.4 \pm 17.6 \mathrm{mmHg}(p=0.04)$. Right atrial pressure, estimated using the diameter of the inferior vena cava, also decreased significantly at follow-up $(p<0.001)$.

Initially patients had RV dilatation that improved significantly after 1 month of therapy $(p=0.03)$. There was no significant difference in mean $\mathrm{RV}$ diameter between controls and the follow-up patients $(p=0.38)$, indicating complete recovery of RV diameter. RV function improved significantly as assessed by the median TAPSE (baseline: 15.2[13.3-16.8] mm vs. follow-up: 23.1 [20.5- 25.6] $\mathrm{mm}, \mathrm{p}<0.001$ ), mean tricuspid peak $\mathrm{s}$ wave (baseline: $10.0 \pm 1.5 \mathrm{~cm} / \mathrm{s}$ vs. follow-up: $12.8 \pm 4.0 \mathrm{~cm} / \mathrm{s}, p=0.007$ ) and RV fractional area change (baseline 34.4 [30.5- 39.8] \% vs. follow-up 39.3 [35.0 - 43.6], $p=0.02$ ). There was no difference between controls and the follow-up patients for these three parameters. The TAPSE/SPAP ratio, an indicator of pulmonary compliance, improves after 1 month in patients. Given the lack of measurable PA pressures in controls, the comparison with controls was not possible.

Left ventricular ejection fraction was normal in patients at the initial phase of PE (mean LVEF $60.2 \pm 7.0 \%$ ) but significantly improved at follow- 
up $(68.4 \pm 6.7 \%, p=0.003)$. In contrast, no difference in $E / e^{\prime}$ ratio (at the lateral mitral annulus) was found.

\section{Three-dimensional RV function}

Table 2 summarizes the evolution of 3D RV echocardiographic parameters.

$\underline{R V \text { volumes and ejection fraction }}$

RV end-diastolic volumes decreased significantly (baseline: $101.3 \pm 28.7 \mathrm{ml}$ vs. follow-up: $76.1 \pm 20.6 \mathrm{ml}, \mathrm{p}<0.001$ ) whereas RV ejection fraction significantly improved (baseline: $30.9 \pm 7.4 \%$ vs. follow-up: $45.8 \pm 6.6 \%, p<0.001)$.

RV end-diastolic volumes were significantly higher in baseline patients than in controls $(101.3 \pm 8.7 \mathrm{ml}$ vs. $66.6 \pm 24.8 \mathrm{ml}, \mathrm{p}<0.001)$. RVEF was also significantly lower in baseline patients compared to controls $(p<0.001)$. In contrast, follow-up patients' volumes were not significantly different from controls' ones $(p=0.24)$. However, RVEF remained lower in follow-up patients compared controls $(45.8 \pm 6.6 \%$ vs $54.3 \pm 7.6 \%$; $\mathrm{p}<0.001)$.

Intra-class correlation coefficients for inter-observer variability were 0.90 [0.77-0.96] for RV EF and 0.94 [0.86-0.98] for RV EDV. Intra- class correlation coefficients for intra-observer variability were 0.95 [0.88-0.98] for RV EF, 0.98 [0.96-0.99] for RV EDV, and 0.93 [0.83-0.97] for RV global AS, respectively.

\section{$\underline{R V \text { strain }}$}

The evolution of strain after PE varied among the strain components: circumferential and area strain were highly impacted at baseline while they recovered at 1 month; in contrast, no difference was observed in longitudinal strain (Table 2).

There were differences in area strain between baseline and followup, but also differences in area strain between controls (who have the greatest strain) and follow-up patients (Figure 2). 
Circumferential strain was impaired at baseline in almost all RV segments. The whole septum as well as the RV apex completely recovered, with no difference between follow-up patients and controls. In the free wall, RV circumferential strain improved at follow-up but remained lower compared to controls (Table 2 ).

There was no significant change in longitudinal strain from baseline to follow-up, except at the apical segment of the septum. However, RV free-wall longitudinal strain was impaired at baseline and remains altered at follow-up as compared to controls. In contrast, given the integrity of the septal longitudinal strain, the global RV longitudinal strain was not significantly different in patients at the follow-up in comparison with controls.

\section{Relationship between RV area strain and the McConnell's sign}

The McConnell's sign was present in $83 \%$ of patients and in none of the controls. Figure 3 illustrates the association between the McConnell's sign, global RV area strain and apical area strain. McConnell's sign was associated with lower RV apical area strain $(p<0.001)$, with an AUC of 0.881 associated to an apical area strain $>-20 \%$. 


\section{DISCUSSION}

This is the first 3D RV speckle-tracking study in acute pulmonary embolism patients. In intermediate-high-risk patients, RV function either assessed by 2D or 3D is impaired in the acute phase compared to a control population. However, 3D RV strain (area and circumferential strain) remains altered at 1 month follow-up, unlike the standard 2D RV function parameters, indicating an incomplete recovery at 1 month after discharge. RV longitudinal strain is less impacted by acute pressure overload than circumferential strain and area strain. The McConnell's sign, described as the preservation of apical contraction, is associated with weaker deformation at the apex and to a broader extent in the right ventricle.

\section{Analysis and interpretation of RV deformation}

The analysis of 2D RV function is limited as it almost only evaluates the regional longitudinal function at the annulus. Indeed, most of RV deformation as assessed in controls comes from the body of the lateral wall and not from the excursion of the tricuspid annulus.

In contrast, 3D quantification tools provide novel insights into these patients. The alteration of 3D longitudinal strain during the acute phase of PE occurs in the RV free wall, not in the septum whereas area and circumferential strain are impaired within the whole RV. Our findings emphasize the need for both global and regional RV assessment, beyond tricuspid annular parameters.

In addition, the impairment of longitudinal strain is less significant than for the circumferential and the area strains, which suggests that even in the presence of a normal TAPSE, RV dysfunction may result from the impairment of the area and circumferential strains.

In our study, 2D RV systolic function appears to be restored at 1 month. Indeed, there is no difference between follow-up patients and controls regarding the TAPSE, the tricuspid valve $s^{\prime}$, the RV dimensions and RV/LV ratio. However, 3D RV assessment leads to different conclusions: area and circumferential strain are still slightly impaired in follow-up patients 
compared to controls, both globally and over the free wall. The incomplete recovery of 3D strain at 1 month indicates a lower degree of recovery (or insufficiently long delay) of patients after PE. The evolution of the strain on the septal wall was subtler, with higher deformation of the controls compared to baseline patients, mainly at the body and apical septum levels. These observations are consistent with the evolution of RVEF at follow-up. The RV strain impairment may be related to persistent pulmonary obstruction or impaired pulmonary compliance post-PE. This is consistent with TAPSE/SPAP ratio that improves after 1-month in our cohort but 1-month follow-up TAPSE/SPAP mean ratio remains abnormal (0.68 \pm 0.25 ) in comparison with published data [11]. This could indicate RV-PA persistent uncoupling in our patients 1-month post-PE [12].

The literature does not report any study of 3D strain in patients hospitalized for pulmonary embolism. 2D strain has been suggested as a significant RV dysfunction parameter in acute PE [13, 14], RV free-wall longitudinal strain being reduced in high-risk patients, however conflicting data exist regarding the significance of 2D RV strain in this situation[15]. This might be related to RV regional dysfunction, misdiagnosed using 2D in comparison with 3D. Our results also suggest that the evaluation of the right ventricle via traditional 2D parameters could be falsely reassuring while abnormalities actually persist, as assessed by 3D strain. This technique is therefore of interest to evaluate RV dysfunction in the acute phase of pulmonary embolism and to investigate persistent symptoms after PE in the absence of pulmonary hypertension. Impaired strain after PE could indicate high risk patients for the development of persistent dyspnoea and functional limitation after acute PE, the "post-PE syndrome" [16]., described in up to $50 \%$ of patients after PE.

\section{McConnell's sign}

Hyperkinesis or normokinesis of the apex is visible in 2D[8] and referred to as the McConnell's sign. However, this visual and subjective assessment has not been assessed quantitatively and objectively, nor related with deformation values. We report that the presence of the 
McConnell's sign correlates with the strain impairment, including at the apex. The visual McConnell's sign refers to a displacement of the apex, pulled by the outflow tract and the left ventricle but not necessarily stands for a preservation of the myocardial deformation of the apex. On the contrary, more severe PE leads to lower RV deformation but more visible McConnell's sign. Similar observations were reported using 2D parameters [17] This apical movement despite a reduced deformation could be explained by the left ventricular apex pulling the RV apex. The left ventricle maintains normal contractility in the context of increasing RV load and decreasing RV function[18].

\section{D speckle-tracking.}

The 3D echocardiographic strain evaluation is highly feasible, reproducible, fast, and more complete than the 2D assessment[19,20,21]. To date, 3D strain has been hardly studied in the RV due to the limited access to relevant commercial software[9, 22, 23]. For similar reasons, few works go beyond global or wall-specific values and compare strain values at the finer regional or local levels[9, 24]. "Normal" values determined in very large populations have not been reported yet, except for the right ventricular morphology (curvature indices)[25]. The prognostic value of RV area strain has already been demonstrated in a cohort of patients with pulmonary hypertension[9] and should also be assessed in PE patients. As the evidence of the usefulness of 3D RV strain assessment grows, the technique development may lead in the next future to automatized software that may allow their use even in the intensive care unit in the context of emergency. 3D strain might be incorporated in a multiparametric algorithm that will help stratify the patient's risk and choose the adequate therapy.

\section{Limitations of the study:}

We only selected patients with intermediate-high risk PE in this preliminary study. This potential selection bias is sustained by the need to investigate patients with RV myocardial damage in whom strain 
differences were expected. Transthoracic echocardiograms with 2D and 3D acquisitions were performed in all patients within the first 12 hours of hospitalization emphasizing the technical difficulty of the study. Furthermore, the method is limited by the availability of 3D echocardiography during such a short time interval. Acute dyspnoea also influenced the technical realization of the examination, with consequences on the inclusion of PE patients. In our study, four patients were excluded because of low acoustic window.

The recruitment of age- and sex-matched controls with no significant heart disease was very demanding. As echogenicity worsens with age, several controls were retrospectively excluded due to insufficient 3D imaging for RV volumes quantification.

One month is a rather short period to evaluate strain recovery over time. Myocardial strain may recover later than this 1 month period, the hypothesis of persistent RV function impairment is strongly suggested despite the normalization of other 2D parameters. Follow-up over a longer period would determine whether the impairment that remains at one month will always persist or is due to this short follow-up period.

\section{CONCLUSION}

After one month of anticoagulant therapy in patients with intermediatehigh risk PE, RV function has not fully recovered as assessed by 3D RV area and circumferential strain, while 2D assessment misses this incomplete recovery.

These persistent abnormalities may indicate either an aftereffect of pulmonary embolism that will persist over time and eventually be associated with chronic post-embolic dyspnoea / chronic post-embolic pulmonary hypertension or a slower recovery of RV myocardial deformation than predicted.

Furthermore, McConnell's sign, a traditionally described sign of pulmonary embolism, is significantly associated with impaired RV apical 
and global strain. Unlike the visual impression, this sign indicates a lower RV strain.

\section{FUNDING SOURCES:}

AO2I 2013 CHU de Nice grant

European Union 7th Framework Program (VP2HF: FP7-2013-611823)

\section{DATA AVAILABILITY STATEMENT:}

Data are available on demand 


\section{REFERENCES}

[1] White $\mathrm{RH}$. The epidemiology of venous thromboembolism. Circulation 2003;107:14-8.

[2] Rudski LG, Lai WW, Afilalo J, et al. Guidelines for the Echocardiographic Assessment of the Right Heart in Adults: A Report from the American Society of Echocardiography. J Am Soc Echocardiogr 2010;23:685-713.

[3] Lang RM, Badano LP, Mor-Avi V, et al. Recommendations for cardiac chamber quantification by echocardiography in adults: an update from the American Society of Echocardiography and the European Association of Cardiovascular Imaging. Eur Heart J Cardiovasc Imaging. 2015;16:233-70.

[4] Friedberg MK. Imaging Right-Left Ventricular Interactions. JACC Cardiovascular imaging 2018;11:755-71.

[5] Muraru D, Niero A, Rodriguez-Zanella $\mathrm{H}$, et al. Three-dimensional speckle-tracking echocardiography: benefits and limitations of integrating myocardial mechanics with three-dimensional imaging. Cardiovasc Diagn Ther 2018;8:101-17.

[6] Konstantinides SV, Torbicki A, Agnelli G, et al. 2014 ESC guidelines on the diagnosis and management of acute pulmonary embolism. Eur Heart J 2014;35:3033-69, 69a-69k.

[7] Douglas PS, DeCara JM, Devereux RB, et al. Echocardiographic Imaging in Clinical Trials: American Society of Echocardiography Standards for Echocardiography Core Laboratories. J Am Soc Echocardiogr 2009;22:755-65.

[8] McConnell MV, Solomon SD, Rayan ME, et al. Regional right ventricular dysfunction detected by echocardiography in acute pulmonary embolism. Am J Cardiol 1996;78:469-73.

[9] Moceri P, Duchateau N, Baudouy D, et al. Three-dimensional rightventricular regional deformation and survival in pulmonary hypertension. Eur Heart J Cardiovasc Imaging 2018;19:450-8.

[10] Weyman AE. Principles and Practice of: Echocardiography. Lea \& Febiger 1994. 
[11] Ferrara F, Rudski LG, Vriz O, et al. Physiologic correlates of tricuspid annular plane systolic excursion in 1168 healthy subjects. Int J Cardiol. 2016;223:736-743.

[12] Guazzi M. Use of TAPSE/PASP ratio in pulmonary arterial hypertension: An easy shortcut in a congested road. Int J Cardiol. 2018;266:242-244.

[13] Descotes-Genon V, Chopard R, Morel M, et al. Comparison of right ventricular systolic function in patients with low risk and intermediate-tohigh risk pulmonary embolism: a two-dimensional strain imaging study. Echocardiography 2013;30:301-8.

[14]Vitarelli A, Mangieri E, Terzano C, et al. Three-Dimensional Echocardiography and 2D-3D Speckle-Tracking Imaging in Chronic Pulmonary Hypertension: Diagnostic Accuracy in Detecting Hemodynamic Signs of Right Ventricular (RV) Failure. J Am Heart Assoc. 2015;4:e001584e.

[15] Khemasuwan D, Yingchoncharoen T, Tunsupon P, et al. Right ventricular echocardiographic parameters are associated with mortality after acute pulmonary embolism. J Am Soc Echocardiogr 2015;28:355-62.

[16] Pugliese SC, Kawut SM. The Post-Pulmonary Embolism Syndrome: Real or Ruse? Ann Am Thorac Soc. 2019;16:811-814.

[17] Platz E, Hassanein AH, Shah A, et al. Regional right ventricular strain pattern in patients with acute pulmonary embolism. Echocardiography 2012;29:464-70.

[18] Naeije R, Badagliacca R. The overloaded right heart and ventricular interdependence. Cardiovasc Res 2017;113:1474-85.

[19] Knight DS, Grasso AE, Quail MA, et al. Accuracy and reproducibility of right ventricular quantification in patients with pressure and volume 
overload using single-beat three-dimensional echocardiography. J Am Soc Echocardiogr 2015;28:363-74.

[20] Leibundgut G, Rohner A, Grize L, et al. Dynamic assessment of right ventricular volumes and function by real-time three-dimensional echocardiography: a comparison study with magnetic resonance imaging in 100 adult patients. J Am Soc Echocardiogr 2010;23:116-26.

[21] Grapsa J, O'Regan DP, Pavlopoulos H, et al. Right ventricular remodelling in pulmonary arterial hypertension with three-dimensional echocardiography: comparison with cardiac magnetic resonance imaging. Eur J Echocardiogr 2010;11:64-73.

[22] Addetia K, Maffessanti F, Yamat M, et al. Three-dimensional echocardiography-based analysis of right ventricular shape in pulmonary arterial hypertension. Eur Heart J Cardiovasc Imaging 2016;17:564-75.

[23] Addetia K, Uriel N, Maffessanti F, et al. 3D Morphological Changes in LV and RV During LVAD Ramp Studies. JACC Cardiovascular imaging 2018;11:159-69.

[24] Smith BC, Dobson G, Dawson D, et al. Three-dimensional speckle tracking of the right ventricle: toward optimal quantification of right ventricular dysfunction in pulmonary hypertension. J Am Coll Cardiol 2014;64:41-51.

[25] Addetia K, Maffessanti F, Muraru D, et al. Morphologic Analysis of the Normal Right Ventricle Using Three-Dimensional EchocardiographyDerived Curvature Indices. J Am Soc Echocardiogr 2018;31:614-23. 


\section{TABLES}

Table 1: Two-dimensional echocardiographic characteristics of the studied population

\begin{tabular}{|c|c|c|c|c|c|c|}
\hline & $\begin{array}{l}\text { Echo } 1 \\
\text { Baseline }\end{array}$ & $\begin{array}{c}\text { Echo } 2 \\
\text { 1-month }\end{array}$ & Controls & $\begin{array}{c}\text { p } \\
\text { E1 vs. } \\
\text { E2 }\end{array}$ & $\begin{array}{c}p \\
\text { El vs. } \\
\text { Control } \\
\text { s }\end{array}$ & $\begin{array}{c}p \\
\text { E2 vs. } \\
\text { Control } \\
\text { s }\end{array}$ \\
\hline $\begin{array}{l}\text { RV diameter, } \\
\text { cm }\end{array}$ & $4.2 \pm 0.6$ & $3.5 \pm 1.0$ & $3.1 \pm 0.5$ & 0.03 & $<0.001$ & 0.38 \\
\hline $\mathrm{RV} / \mathrm{LV}$ ratio & $1.1 \pm 0.2$ & $0.8 \pm 0.3$ & $0.6 \pm 0.1$ & $\begin{array}{c}<0.00 \\
1\end{array}$ & $<0.001$ & 0.06 \\
\hline TAPSE, mm & 15.2 [13.3-16.8] & $\begin{array}{l}23.1[20.5- \\
25.6]\end{array}$ & $\begin{array}{l}26.2[22.8- \\
29.9]\end{array}$ & 0.005 & $<0.001$ & 0.10 \\
\hline TV s', cm/s & $10.0 \pm 1.5$ & $12.8 \pm 4.0$ & $13.9 \pm 2.2$ & 0.007 & $<0.001$ & 0.42 \\
\hline RV FAC, \% & $\begin{array}{c}34.4[30.5- \\
39.8]\end{array}$ & $\begin{array}{c}39.3[35.0- \\
43.6]\end{array}$ & $\begin{array}{c}41.2[36.1 \text { - } \\
45.1]\end{array}$ & 0.02 & 0.01 & 0.67 \\
\hline sPAP, $\mathbf{m m H g}$ & $52.2 \pm 18.5$ & $41.4 \pm 17.6$ & $28.1 \pm 10.8$ & 0.04 & & \\
\hline $\begin{array}{l}\text { TAPSE/sPAP, } \\
\mathrm{mm} / \mathrm{mmHg}\end{array}$ & $0.32 \pm 0.13$ & $0.68 \pm 0.25$ & & 0.001 & & \\
\hline LVEF, \% & $60.2 \pm 7.0$ & $68.4 \pm 6.7$ & $66.0 \pm 7.1$ & 0.003 & 0.01 & 0.31 \\
\hline E/e' lat & $7.4 \pm 2.6$ & $7.1 \pm 2.6$ & $8.1 \pm 3.3$ & 0.65 & 0.78 & 0.85 \\
\hline $\begin{array}{l}\text { IVC diameter, } \\
\text { mm }\end{array}$ & $20.7 \pm 4.6$ & $12.6 \pm 6.5$ & $14.2 \pm 3.1$ & $\begin{array}{c}<0.00 \\
1\end{array}$ & $<0.001$ & 0.76 \\
\hline
\end{tabular}

FAC, fractional area change; LV, left ventricular; LVEF, left ventricular ejection fraction; RV, right ventricular; SPAP, systolic pulmonary artery pressure; TV, tricuspid valve. 
Table 2: Three-dimensional echocardiographic parameters

\begin{tabular}{|c|c|c|c|c|c|c|}
\hline & $\begin{array}{l}\text { Echo } 1 \\
\text { Baseline }\end{array}$ & $\begin{array}{l}\text { Echo } 2 \\
1 \text { month }\end{array}$ & Controls & $\begin{array}{c}\text { p } \\
\text { E1 vs. } \\
\text { E2 }\end{array}$ & $\begin{array}{c}\text { p } \\
\text { E1 vs. } \\
\text { Controls }\end{array}$ & $\begin{array}{c}p \\
\text { E2 vs. } \\
\text { Control } \\
s\end{array}$ \\
\hline 3D RV EDV, ml & $\begin{array}{c}101.3 \pm \\
28.7\end{array}$ & $76.1 \pm 20.6$ & $66.6 \pm 24.8$ & $\begin{array}{c}0.000 \\
4\end{array}$ & 0.0005 & 0.24 \\
\hline 3D RV EF, \% & $30.9 \pm 7.4$ & $45.8 \pm 6.6$ & $54.3 \pm 7.6$ & $\begin{array}{c}<0.00 \\
01\end{array}$ & $<0.0001$ & 0.0009 \\
\hline \multicolumn{7}{|l|}{ RV Area strain, \% } \\
\hline Global & $-17.3 \pm 5.4$ & $-23.5 \pm 5.4$ & $-28.7 \pm 5.5$ & 0.003 & $<0.0001$ & 0.004 \\
\hline Apex sep & $\begin{array}{c}-10.0 \pm \\
13.3\end{array}$ & $-21.7 \pm 8.2$ & $-21.8 \pm 9.3$ & 0.001 & 0.002 & 0.95 \\
\hline Body sep & $-19.3 \pm 7.8$ & $-24.3 \pm 8.1$ & $-25.4 \pm 6.7$ & 0.017 & 0.009 & 0.64 \\
\hline Inlet sep & $\begin{array}{c}-13.1 \pm \\
20.2\end{array}$ & $-14.8 \pm 9.4$ & $\begin{array}{c}-21.5 \pm \\
10.0\end{array}$ & 0.07 & 0.08 & 0.03 \\
\hline Outlet sep & $-18.9 \pm 8.4$ & $-15.5 \pm 16.3$ & $\begin{array}{l}-22.6 \pm \\
13.1\end{array}$ & 0.47 & 0.30 & 0.13 \\
\hline Apex lat & $-15.6 \pm 6.9$ & $-24.5 \pm 7.2$ & $-28.0 \pm 7.9$ & 0.002 & $<0.0001$ & 0.15 \\
\hline Body lat & $-21.3 \pm 6.5$ & $-30.2 \pm 6.1$ & $-36.5 \pm 7.0$ & 0.001 & $<0.0001$ & 0.004 \\
\hline Inlet lat & $-19.9 \pm 5.4$ & $-26.5 \pm 5.9$ & $-35.0 \pm 7.6$ & 0.003 & $<0.0001$ & 0.0003 \\
\hline Outlet lat & $-10.7 \pm 3.7$ & $-13.9 \pm 5.8$ & $-17.2 \pm 4.6$ & 0.10 & $<0.0001$ & 0.04 \\
\hline RV free wall & $-16.9 \pm 4.3$ & $-23.8 \pm 5.1$ & $-29.2 \pm 5.3$ & 0.001 & $<0.0001$ & 0.002 \\
\hline \multicolumn{7}{|l|}{$\begin{array}{l}\text { RV Longitudinal } \\
\text { strain,\% }\end{array}$} \\
\hline Global & $-8.8 \pm 3.4$ & $-11.6 \pm 6.3$ & $-13.7 \pm 4.0$ & 0.15 & 0.0001 & 0.19 \\
\hline Apex sep & $-4.8 \pm 11.3$ & $-11.9 \pm 7.6$ & $-10.9 \pm 8.9$ & 0.002 & 0.06 & 0.72 \\
\hline Body sep & $-10.9 \pm 5.6$ & $-13.1 \pm 6.6$ & $-14.1 \pm 6.1$ & 0.27 & 0.09 & 0.59 \\
\hline Inlet sep & $-8.2 \pm 6.3$ & $-4.8 \pm 9.4$ & $-12.4 \pm 8.4$ & 0.22 & 0.08 & 0.009 \\
\hline Outlet sep & $-6.2 \pm 7.7$ & $\begin{array}{c}-10.93 \pm \\
15.1\end{array}$ & $-9.1 \pm 10.1$ & 0.11 & 0.30 & 0.02 \\
\hline Apex lat & $-8.6 \pm 4.4$ & $-11.4 \pm 6.1$ & $-14.0 \pm 5.0$ & 0.14 & 0.0006 & 0.13 \\
\hline Body lat & $-9.5 \pm 5.5$ & $-11.7 \pm 5.2$ & $-14.2 \pm 6.7$ & 0.27 & 0.02 & 0.19 \\
\hline Inlet lat & $-11.5 \pm 4.3$ & $-14.0 \pm 3.9$ & $-18.3 \pm 5.8$ & 0.07 & 0.0002 & 0.01 \\
\hline Outlet lat & $-4.5 \pm 2.8$ & $-5.8 \pm 4.0$ & $-8.3 \pm 3.2$ & 0.31 & 0.0003 & 0.03 \\
\hline RV free wall & $-8.5 \pm 3.0$ & $-10.7 \pm 3.8$ & $-13.7 \pm 3.5$ & 0.07 & $<0.0001$ & 0.01 \\
\hline \multicolumn{7}{|l|}{$\begin{array}{l}\text { RV Circumferential } \\
\text { strain,\% }\end{array}$} \\
\hline Global & $-9.2 \pm 3.4$ & $-14.1 \pm 3.3$ & $-16.8 \pm 3.7$ & $\begin{array}{c}0.000 \\
6\end{array}$ & $<0.0001$ & 0.02 \\
\hline Apex sep & $-4.7 \pm 5.7$ & $-11.1 \pm 4.2$ & $-12.0 \pm 4.8$ & $\begin{array}{c}0.000 \\
6\end{array}$ & 0.0001 & 0.52 \\
\hline Body sep & $-7.9 \pm 5.6$ & $-11.6 \pm 4.2$ & $-11.9 \pm 3.3$ & 0.02 & 0.006 & 0.82 \\
\hline Inlet sep & $-9.0 \pm 6.3$ & $-10.1 \pm 5.7$ & $-10.0 \pm 6.6$ & 0.45 & 0.63 & 0.94 \\
\hline Outlet sep & $-12.9 \pm 7.3$ & $-13.9 \pm 8.6$ & $-13.2 \pm 5.6$ & 0.74 & 0.90 & 0.76 \\
\hline Apex lat & $-7.5 \pm 4.8$ & $-14.8 \pm 4.0$ & $-16.5 \pm 5.6$ & $\begin{array}{c}0.000 \\
3\end{array}$ & $<0.0001$ & 0.27 \\
\hline Body lat & $-12.4 \pm 5.1$ & $-20.2 \pm 5.5$ & $-24.9 \pm 4.6$ & 0.001 & $<0.0001$ & 0.004 \\
\hline Inlet lat & $-9.1 \pm 3.1$ & $-13.7 \pm 4.2$ & $-19.3 \pm 6.6$ & 0.001 & $<0.0001$ & 0.003 \\
\hline Outlet lat & $-6.4 \pm 2.8$ & $-8.4 \pm 3.0$ & $-10.0 \pm 3.1$ & 0.05 & 0.0004 & 0.10 \\
\hline RV free wall & $-8.8 \pm 3.1$ & $-14.3 \pm 3.3$ & $-17.7 \pm 4.1$ & $\begin{array}{c}0.000 \\
2\end{array}$ & $<0.0001$ & 0.006 \\
\hline
\end{tabular}




\section{FIGURES LEGENDS}

Figure 1: Right ventricular segmentation used in our study.

Figure 2: Area strain in patients and controls represented over the mean RV shape of each group: The 1st line represents the septum whereas the $2^{\text {nd }}$ line represents the RV free wall. The color code encodes the median area strain at each point of the RV mesh in PE patients on admission (1st column), 1 month after their hospitalization ( 2 nd column), and in controls (4th column), and the differences between baseline and follow-up (3rd column, where blue color stands for more marked and namely improving deformation).

Figure 3: Relationship between the McConnell's sign and RV area strain. On the right side of the panel differences in RV apical and global strain in patients with or without the Mc Connell's sign. On the left, the ROC curve illustrates the association between the Mc Connell's sign and impaired apical area strain (RV apical area strain $>-20 \%$ ). 
Figure 1

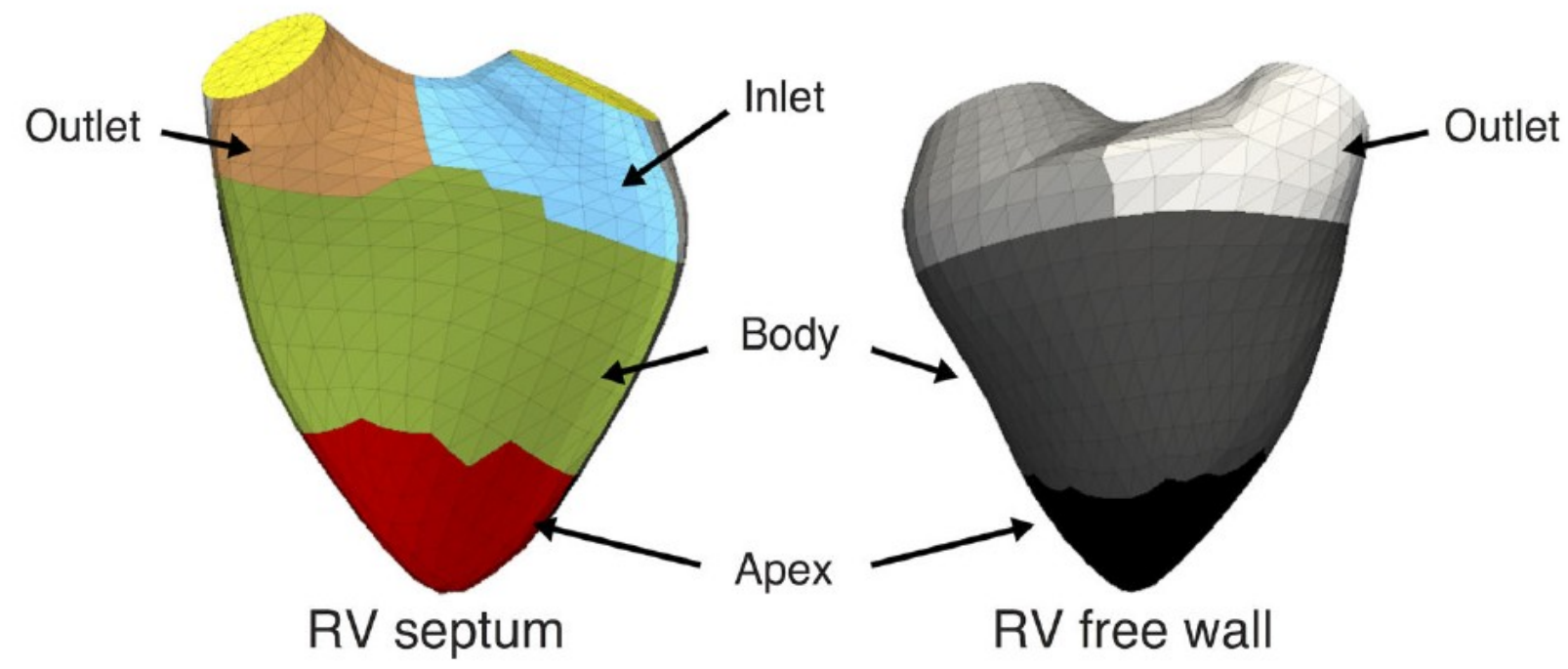


Figure 2

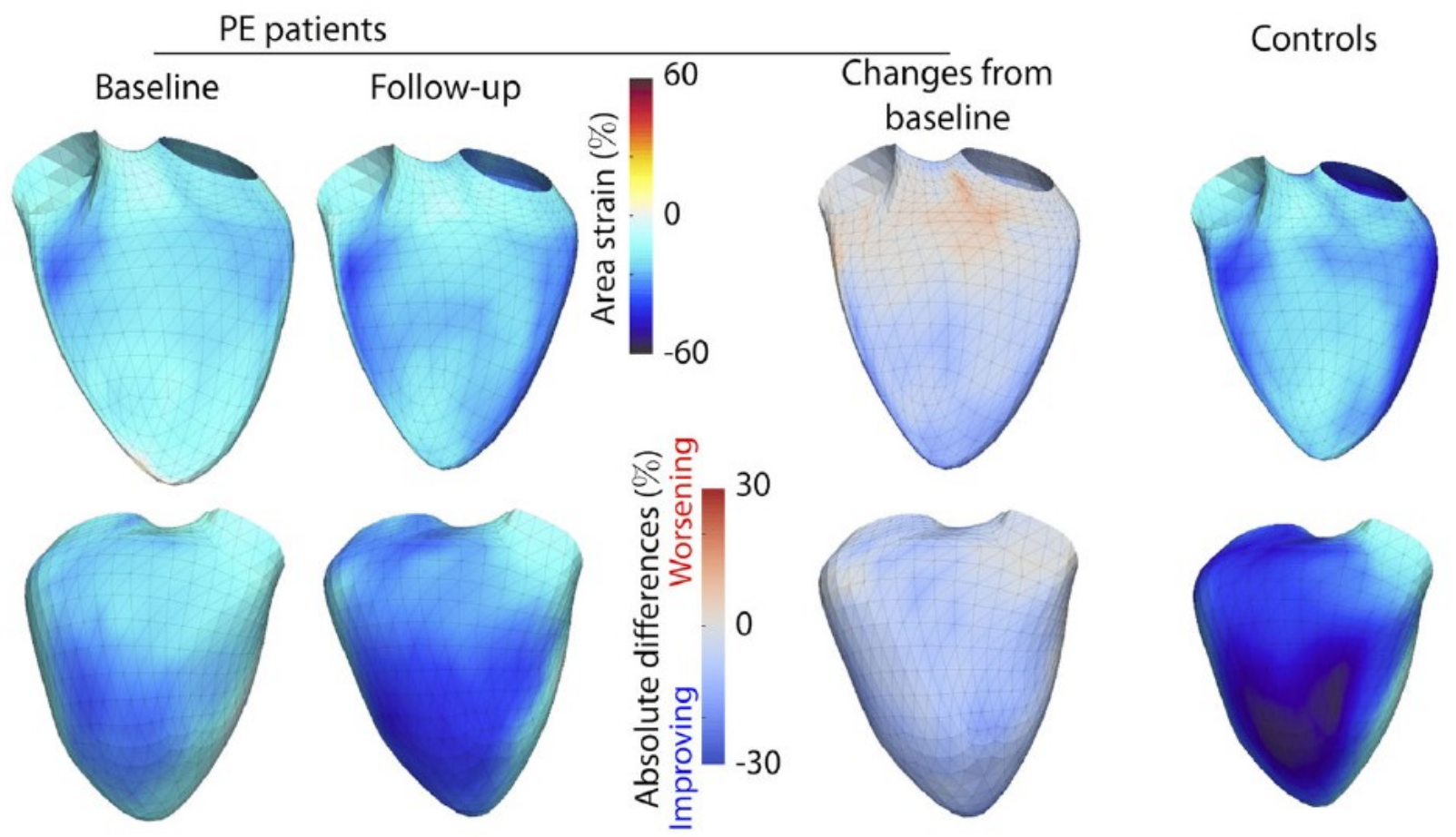


Figure 3
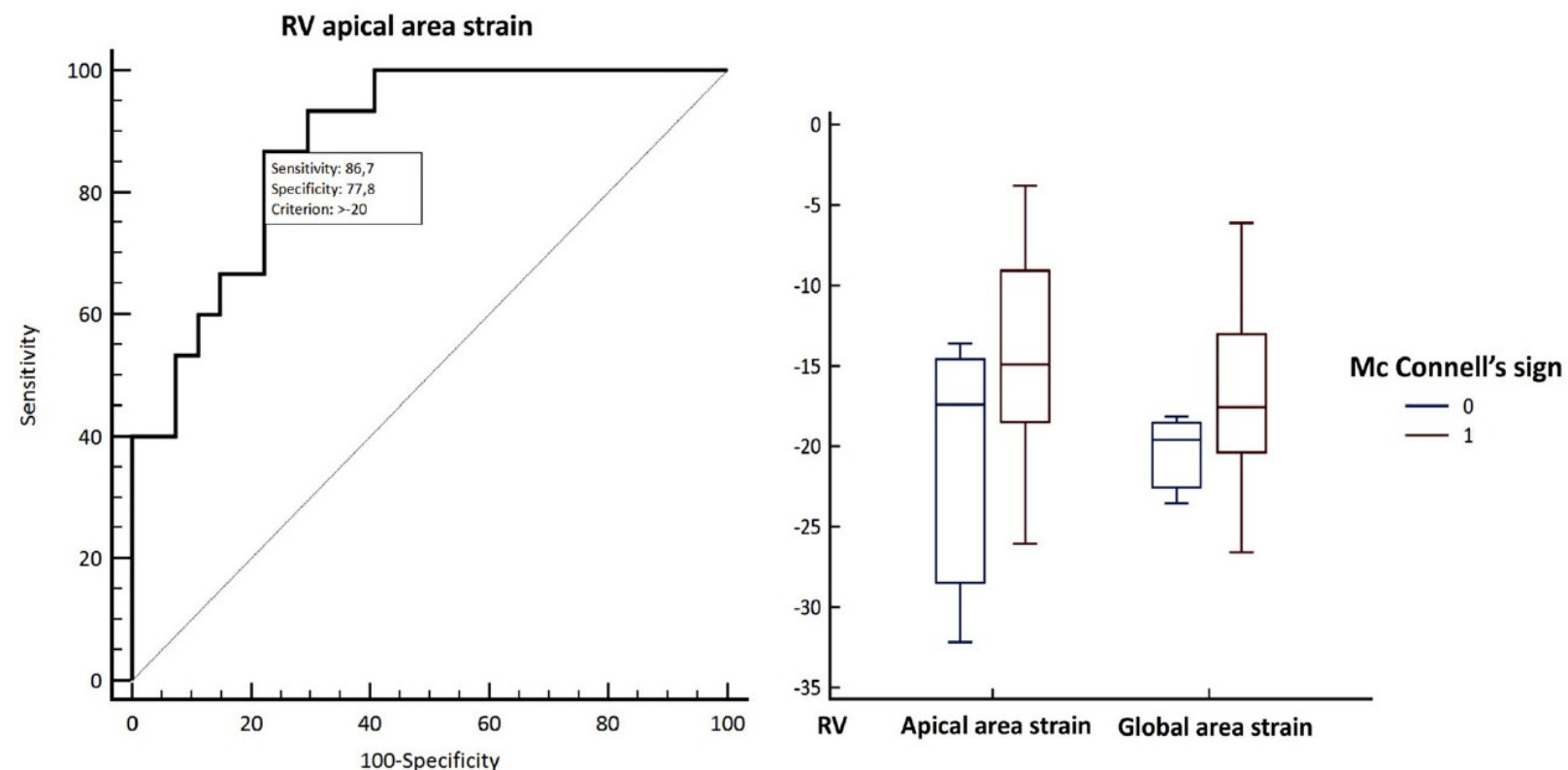